\title{
VLSI Implementation of Segmentation of Single Channel ECG
}

\author{
S. Preethi \\ PG Scholar \\ Kalaignar Karunanidhi Institute of Technology \\ Coimbatore, India.
}

\author{
M. Jayasheela \\ $\mathrm{HOD} / \mathrm{PG}$ \\ Kalaignar Karunanidhi Institute of Technology \\ Coimbatore, India.
}

\begin{abstract}
:
In this paper the implementation of extraction of respiratory signal from ECG signal in very large scale integration (VLSI) using discrete wavelet transform (DWT) is discussed. The Indirect method to obtain the respiratory signal from ECG can benefit both respiratory and cardiac activities. The accuracy of the extracted respiratory signal will be high. The implementation is based on bit serial approach (BS) which substitute multiply and accumulate operations. BS provides a multiplication-free method for calculating inner products of fixed-point data. But increase in filter order leads complexity. Here we propose high-speed and low power architecture. The proposed method is implemented in hardware description language and verified via simulation. The proposed architecture scheme yields significantly reduced complexity, less area and high speed features.
\end{abstract}

\section{Keywords}

Finite impulse response (FIR), bit serial approach (BS), discrete wavelet transforms (DWT), very large scale integration (VLSI).

\section{INTRODUCTION}

The respiratory signal is normally recorded in spirometry; however this cannot be used in cases like sleep studies and stress testing. So the respiratory signal is extracted from the ECG signal. ECG is recorded from the upper surface of the heart by an electrode which accompanies the respiration results like expansion and contraction of the chest.

The advantage of this method is that low cost, high efficiency and both the cardiac and respiratory activities are monitored. Wavelet Transform (WT) is broadly used, which is a fundamental tool in pre-processing and characterization procedures. Therefore, in this section, the definition, types and multi resolution analysis of WT are briefly analyzed.

Wavelet analysis provides information that is localized in frequency and in time, which makes it highly suitable for analysis of non-stationary signals and applications in bio signal analyzes and data compression and signal separation. Finite impulse response (FIR) digital filters are common components in many digital signal processing (DSP) [2-11] systems.

Throughout the years, with the increasingly development in very large scale integration (VLSI) technology, the real time realization of FIR filter with less hardware requirement and less latency has become more and more important. Because the complexity of implementation grows with the length of filter, several algorithms have been made to develop effective architectures for realization of FIR filters in application specific integrated circuits (ASIC) and field programmable gate arrays (FPGA) platforms and one of them is bit serial approach(BS).

Moreover, this technology represents a number of attractive features such as simplicity, regularity and modularity of architecture. In recent years, BS-based FIR filter has gained substantial popularity as a primary DSP operation and are rapidly replacing classic analog filters.

The paper is organized as follows: in the next section the algorithm of the dwt is explained then in, section 3 the various processes in DWT and the extraction process are underlined, the obtained results are discussed. Conclusive remarks are given in Section 4.

\section{WAVELET ANALYSIS}

Wavelet analysis gives a time-scale representation for a onedimensional signal. The frequency localization is logarithmic, so the time localization gets finer in the highest frequency range. The wavelet transform (WT) decomposes the signal on a set of bases functions. These bases functions are called as the wavelets. The basis vector is obtained through a family of functions dependent on two parameters: the dilatation (scale) coefficient $(a)$ and the translation step $(b)$. The single prototype wavelet function $\psi(t)$, is stretched or compressed and translated in order to recover the original signal. Since no specific scale is privileged, the wavelet analysis is self-similar at all scales.

The WT of a signal $x(t)$ is defined as:

$$
W_{a} \mathrm{X}(t)=\frac{1}{\sqrt{a}} \int_{-\infty}^{+\infty} x(t) \varphi \frac{t-b}{a} \mathrm{dt}, \quad \mathrm{a}>0
$$

As scale increases, the basis function are dilated; hence, the corresponding coefficients give information about low frequency components of the signal, and when the basis functions are translated the corresponding coefficients give information about the high frequency components of the signal.

When the signal is decomposed the output of the filter are the approximated coefficients $\mathrm{Y}_{\text {low }}$, and detailed coefficients $\mathrm{Y}_{\text {high }}$. 


$$
\begin{aligned}
& \mathrm{Y}_{h i g h}(\mathrm{n})=\sum_{k=-\infty}^{+\infty} x(k) h(2 n-k) \\
& \mathrm{Y}_{\text {low }}(\mathrm{n})=\sum_{k=-\infty}^{+\infty} x(k) g(2 n-k)
\end{aligned}
$$

The DWT computational cost is of the same order of the finite sequence, i.e., $O(N)$, where $N$ is the length of the finite sequence in input [12]. The DWT can be used to extract the RS from ECG signal by selecting a frequency interval on an appropriate level of decomposition.

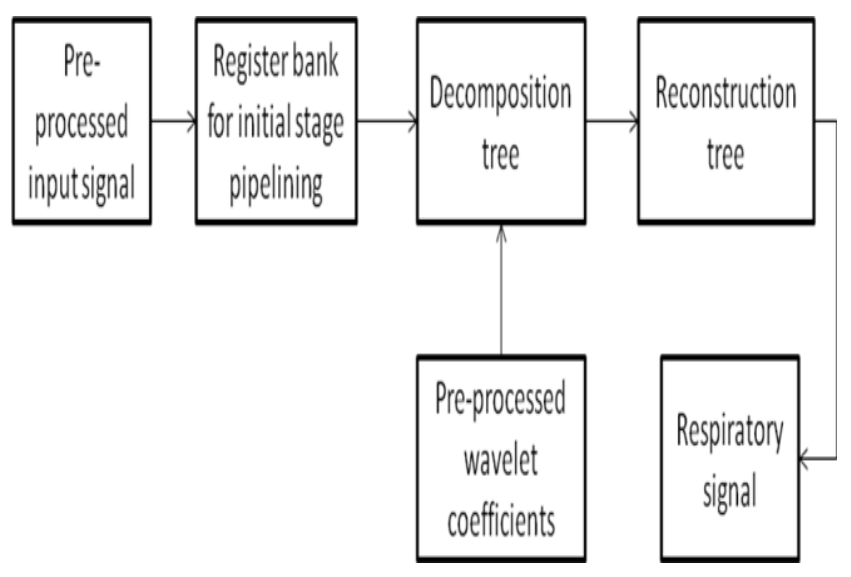

Fig 1: Block Diagram to extract respiratory signal

\section{SIMULATIONS AND EXPERIMENTAL RESULTS}

\subsection{DWT}

The wavelet provides the decomposition of a signal into different time-scales. A test signal is built as the sum of real ECG signal and it is decomposed using DWT. The approximate and detailed coefficients are obtained by decomposing the signal using low pass and high pass filter. The decomposed signals will be verified using filter and the signal in the required frequency range will be filtered and reconstructed. The reconstructed signal will be the residue of the original ECG signal, which is the respiratory signal.

\subsection{Data Pre-processing}

The input given will be in the analog form. In VLSI only the digital form of data's are used as inputs. So the ECG signal in the analog is converted into digital using MATLAB. The wavelet coefficients of the various decomposed levels are also obtained from MATLAB. This is the pre-processed signal which is given as the input signal.

\subsection{Filter Design}

For all decomposition level one low pass and one high pass filer is used. The output of the low pass filter is the approximation signal and the output of the high pass filter is the detailed signal. Both the high pass and low pass filter are designed in Matlab using the Filter Analysis Tool. Using this tool the $\mathrm{H}$ values and the $\mathrm{L}$ values are obtained. The pipelining register is used to buffer the input signal bit by bit and gives it to the decomposition tree. Inside the decomposition tree, the wavelet decomposition is done. Initially, this three level decomposition is done to segment the input ECG signal into six segments.

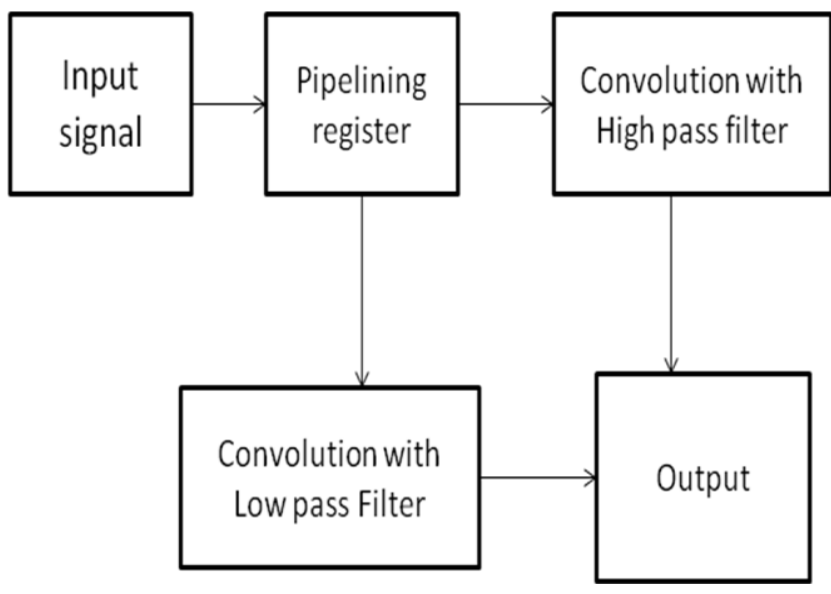

Fig 2: Block diagram for high pass and low pass filter

\subsection{Decomposition}

The original signal entered into decomposition tree will be decomposed into various levels. In level one the signal will be decomposed into approximation and detailed. In level two both the approximation and detailed of level one will again be decomposed into level two approximation and detailed. This process will be continued till the required decomposition level is obtained. From the various levels the required level is selected. This selected signal will be reconstructed. This reconstructed signal is the respiratory signal.

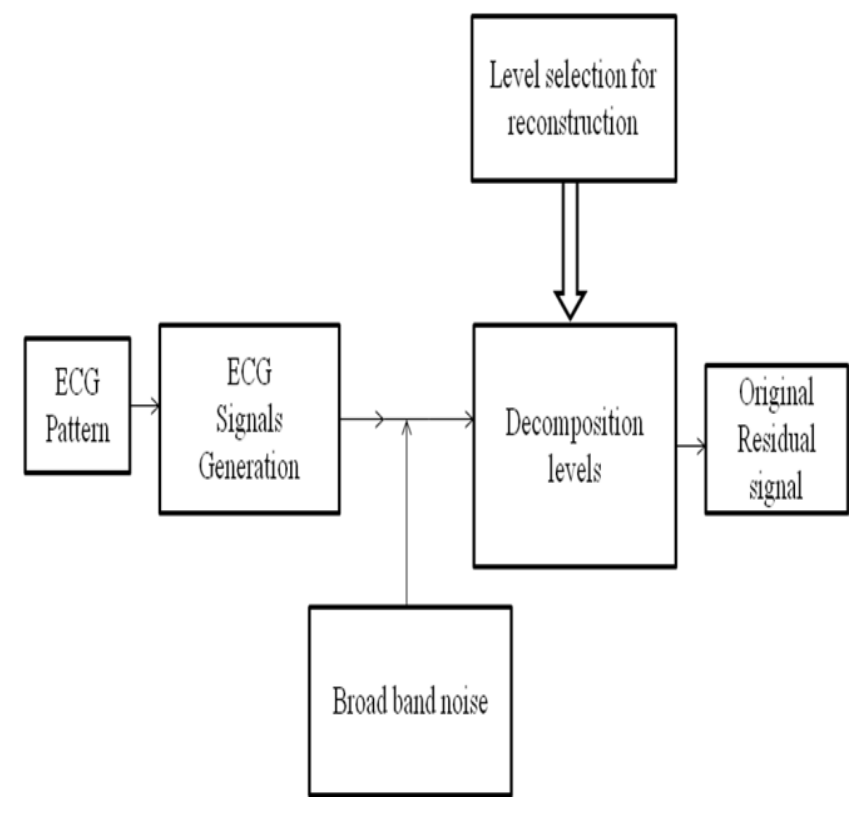

Fig 3: Block diagram for decomposition levels

The below shown fig 4 and fig 5 are obtained from the Quartus II tool which shows the various decomposition levels and the area utilization respectively. The fig 6 is obtained from the modelsim which shows the simulated output for the wavelet decomposition. 


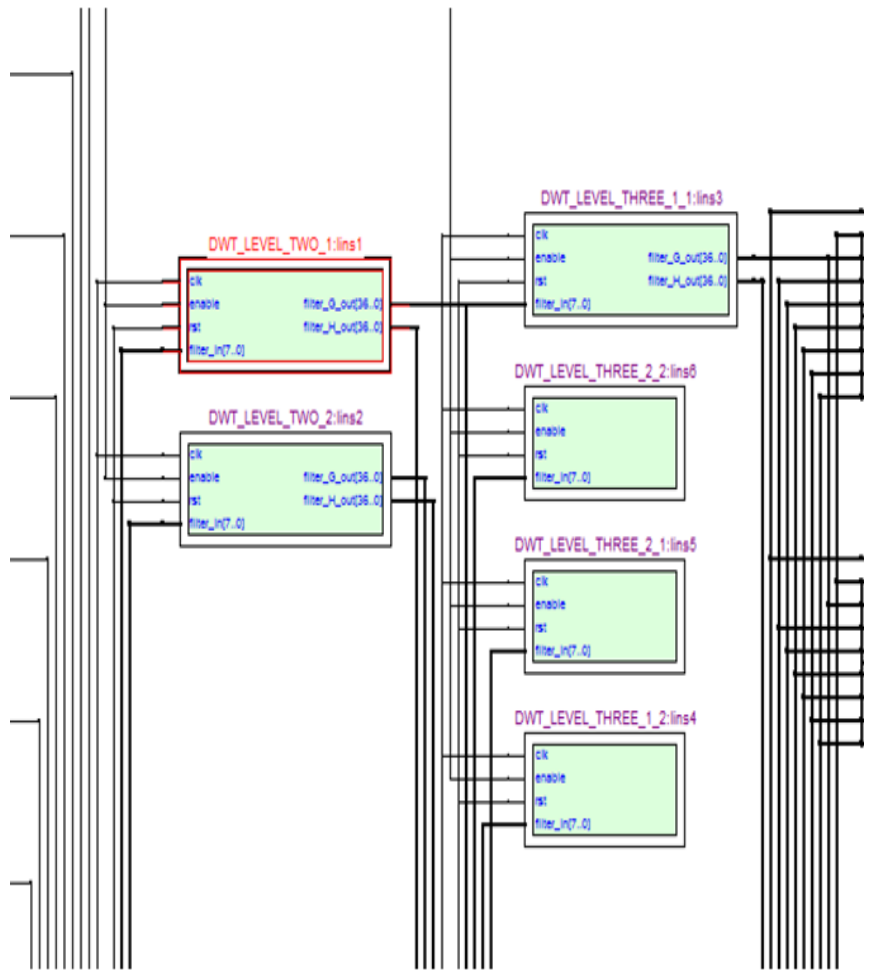

Fig 4: Decomposition levels

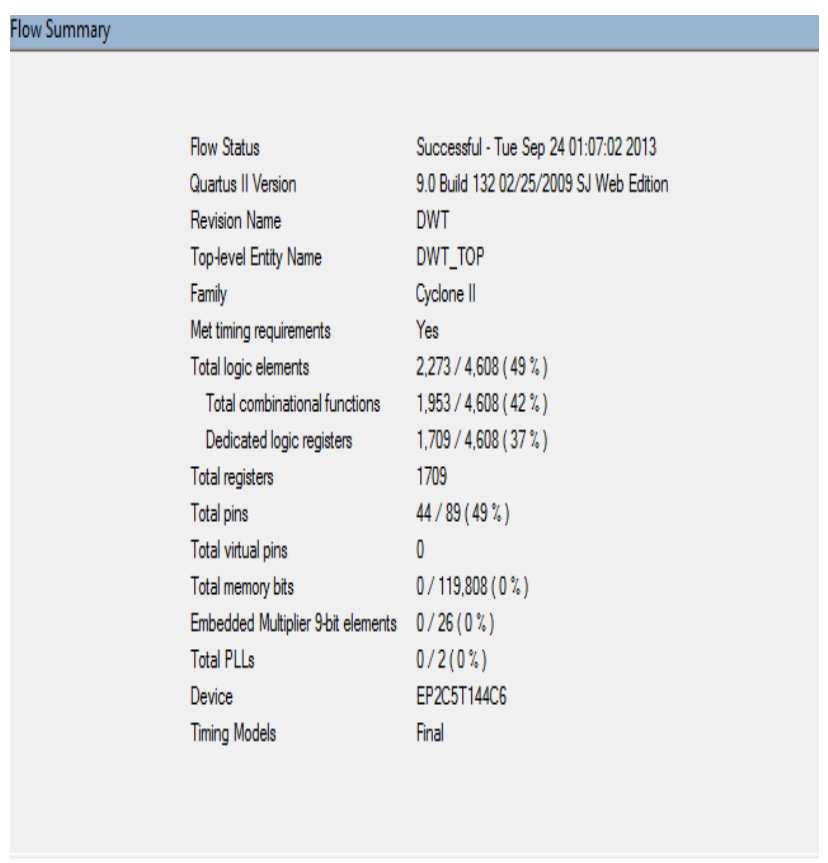

Fig 5: Area utilization report

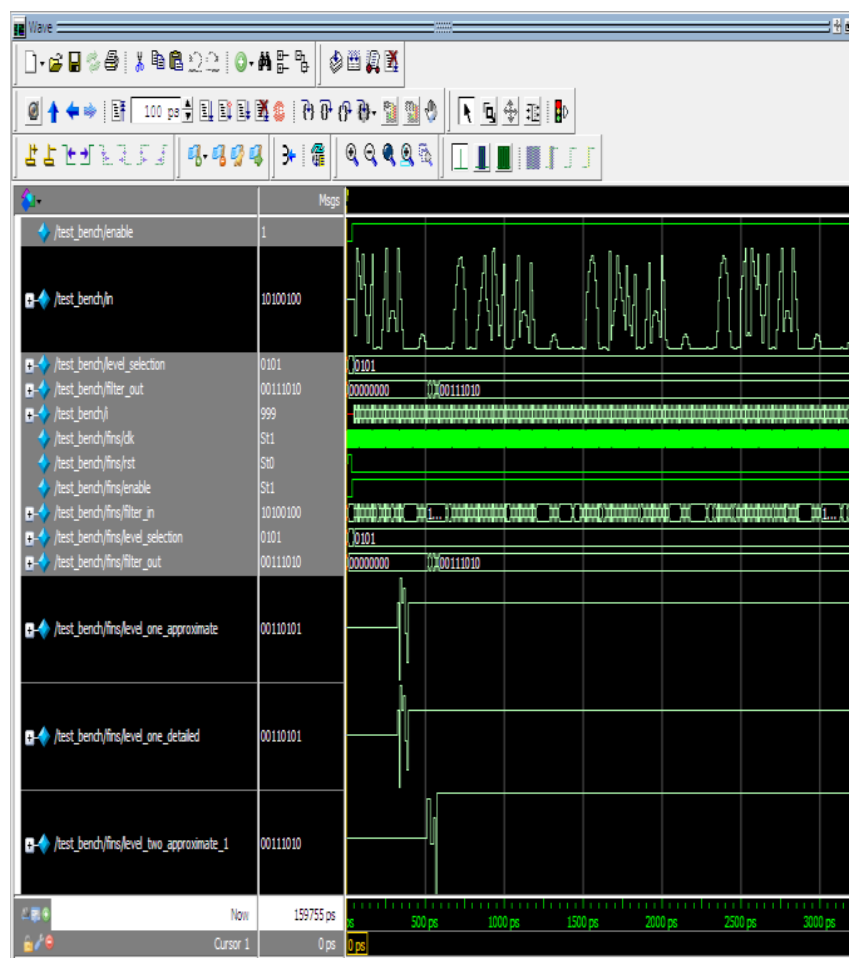

Fig 6: Wavelet Decomposition

\section{CONCLUSION}

In this paper the discrete wavelet transform is used to extract the repiratory signal from the single channel ECG using the verilog which is the hardware description language. Since the bit serial approah is used the speed is increased and power reduced architecture is obtained. This method also reduces the complexity. The input ECG signal is segmented into six signals using three decomposition levels. In each level the detailed and approximate signal of the input signal are obtained. The future work is to reconstruct the respiratory signal. This reconstruction is done by identifying the required respiratory signal from the segmented signal of the ECG signal by using the three level of decomposition.

\section{REFERENCES}

[1] Domenico Labate, Fabio La Foresta, Gianluigi Occhiuto, Francesco Carlo Morabito, Aime Lay-Ekuakille, and Patrizia Vergallo. July 2013. Empirical Mode Decomposition vs. Wavelet Decomposition for the Extraction of Respiratory Signal from Single-Channel ECG: A Comparison, IEEE SENSORS JOURNAL, VOL. 13, NO. 7.

[2] Kung.H.T 1982, Why systolic architecture? IEEE Computer 15, 37-45.

[3] Yu.S and Swartzlander.E.E. 2001. DCT implementation with distributed arithmetic, IEEE Transactions on Computers 50 (9) 985-991.

[4] Hanho Lee and Gerald E. Sobelman. 2002. FPGA-based digit-serial CSD FIR filter for image signal format conversion, Microelectronics Journal 33 (5-6) 501-508.

[5] Valeria Garofalo. 2008. Fixed-width multipliers for the implementation of efficient digital FIR filters, Microelectronics Journal 39 (12) 1491-1498.

[6] Lei Zhang and Tadeusz Kwasniewski. 2009 FIR filter optimization using bit-edge equalization in high-speed 
backplane BSta transmission, Microelectronics Journal 40 (10) 1449-1457.

[7] Eshtawie.M.A.M and Othman.M. 2006 On-line BS-LUT architecture for high-speed high-order digital FIR filters, in: Proceedings of the IEEE International Conference on Communication Systems (ICCS), Singapore.

[8] Choi.J.P, Shin.S.C, and Chung.J.G. 2000 Efficient ROM size reduction for distributed arithmetic, in: Proceedings of the IEEE International Symposium Circuits Systems (ISCAS), pp. 61-64.

[9] Sanjay, Attri B. S, Sohi, and Chopra.Y.C. 2001. Efficient design of application specific DSP cores using FPGAs, in: International Conference on ASIC Proceedings, pp. 462-466.

[10] Kim Kyung-Saeng and Kwyro Lee. 2003 Low-power and area efficient FIR filter implementation suitable for multiple tape, IEEE Transactions on VLSI Systems 11 (1).

[11] Antonion.A. 1993 Digital Filters: Analysis, Design, and Applications, McGraw-Hill, New York.

[12] Labate.D, La Foresta.F, Inuso.G, and Morabito.F.C. 2011 "Remarks about Wavelet Analysis in the EEG Artifacts Detection," in Frontiers in Artificial Intelligence and Application, vol. 226. Amsterdam, The Netherlands: IOS Press, pp. 99-106.

[13] Mallat.S. 1999. A Wavelet Tour of Signal Processing. New York, NY, USA: Academic Press.

[14] Percival.D.B and Walden.A.D. 2000 Wavelet Methods for Time Series Analysis. Cambridge, U.K.: Cambridge Univ. Press. 